\title{
Pentacene in 1,3,5-Tri(1-naphtyl)benzene: A Novel Standard for Transient EPR Spectroscopy at Room Temperature
}

\author{
Mirjam Schröder ${ }^{1,2} \cdot$ Daniel Rauber ${ }^{1}$. Clemens Matt ${ }^{1}$. \\ Christopher W. M. Kay ${ }^{1,3}$ (D)
}

Received: 31 May 2021 / Revised: 15 August 2021 / Accepted: 16 August 2021 / Published online: 7 September 2021

(c) The Author(s) 2021

\begin{abstract}
Testing and calibrating an experimental setup with standard samples is an essential aspect of scientific research. Single crystals of pentacene in $p$-terphenyl are widely used for this purpose in transient electron paramagnetic resonance (EPR) spectroscopy. However, this sample is not without downsides: the crystals need to be grown and the EPR transitions only appear at particular orientations of the crystal with respect to the external magnetic field. An alternative host for pentacene is the glassforming 1,3,5-tri(1-naphtyl)benzene (TNB). Due to the high glass transition point of TNB, an amorphous glass containing randomly oriented pentacene molecules is obtained at room temperature. Here we demonstrate that pentacene dissolved in TNB gives a typical "powder-like" transient EPR spectrum of the triplet state following pulsed laser excitation. From the two-dimensional data set, it is straightforward to obtain the zero-field splitting parameters and relative populations by spectral simulation as well as the $B_{1}$ field in the microwave resonator. Due to the simplicity of preparation, handling and stability, this system is ideal for adjusting the laser beam with respect to the microwave resonator and for introducing students to transient EPR spectroscopy.
\end{abstract}

Prof. Dr. Christopher W. M. Kay would like to thank Prof. Dr. Klaus Möbius for giving him the chance to work at the Institut für Experimentalphysik at the Freie Universität Berlin from 1993 to 2005 .

Christopher W. M. Kay

christopher.kay@uni-saarland.de; c.kay@ucl.ac.uk

1 Department of Chemistry, Saarland University, 66123 Saarbrücken, Germany

2 Present Address: Leibniz-Institut für Katalyse e. V., Albert-Einstein-StraSSe 29a, 18059 Rostock, Germany

3 London Centre for Nanotechnology, University College London, London WC1E 6BT, UK 


\section{Introduction}

The combination of laser excitation and electron paramagnetic resonance (EPR) spectroscopy is a powerful approach for the detection of paramagnetic intermediates. Transient or time-resolved EPR (TREPR) [4, 14, 41] is, therefore, regularly employed in a growing number of laboratories to detect short-lived states including triplets, quintets [35] and radical-pairs [6] in, for example, proteins [43], materials for organic electronics [5, 42] or quantum information science [27]. In the field of dipolar spectroscopy and double electron-electron resonance (DEER) photoexcited triplet states offer the possibility of measuring distances in proteins rather than using conventional nitroxide spin-labels (e.g., (2,2,6,6-Tetramethylpiperidin-1-yl)oxyl, TEMPO) [2, 3]. From these techniques, further highly specific experimental methods can be derived. Magnetophotoselection [26, 33, 39], for example, allows the investigation of the supermolecular arrangement of molecules and hyperfine spectroscopy [38] can be used to investigate the supramolecular interactions within a molecule. The underlying triplet-state physics and an introduction into TREPR instrumentation and spectrum analysis is given in literature [41].

Subsequent to the initial demonstration of TREPR spectroscopy in crystals of fluorene doped with phenazine [17], the triplet state of pentacene diluted in single crystals of $p$-terphenyl (see Fig. 1 for molecular structures) was observed following pulsed laser excitation [18]. The kinetic traces derived from the TREPR experiments are often used to characterize the performance of the EPR spectrometer. For example, the conversion of incident microwave power, $P_{\mathrm{MW}}$, to $B_{1}$-field [19] in the microwave resonator may be determined by analyzing the Torrey oscillations $[36,40]$, which are also referred to as transient nutations [41]. Knowledge of $B_{1}$ is crucial for obtaining a quantitative comparison of samples, since it depends the resonator's bandwidth, $\Delta v$; and thus its $Q$ value which in turn is affected by the dielectric properties of the sample in addition to $P_{\mathrm{MW}}$. These parameters are connected via the conversion efficiency $C^{\prime}$ of a resonator according to Eq. 1 [13].

$$
B_{1}=\frac{C^{\prime} \sqrt{P_{\mathrm{MW}}}}{\sqrt{\Delta v}} .
$$
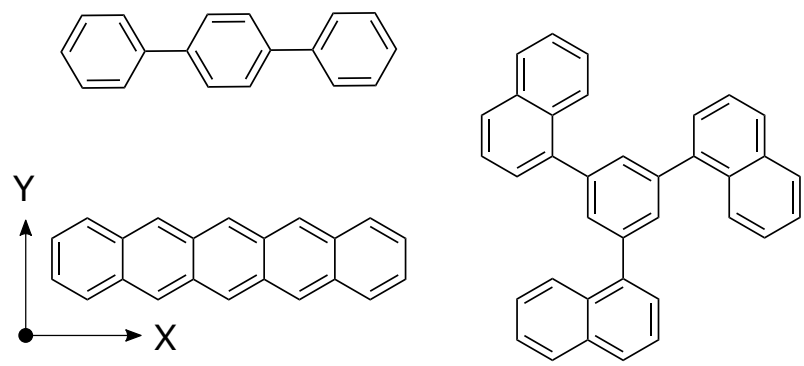

Fig. 1 Structures of pentacene with its main axes (left, bottom) and TNB (right) and p-terphenyl (left, top) 
Alternatively, the conversion factor $C$ directly converts the incident microwave power to $B_{1}$ field without reference to the bandwidth according to Eq. 2:

$$
B_{1}=C \cdot \sqrt{P_{\mathrm{MW}}}
$$

Due to low solubility of pentacene in $p$-terphenyl, the maximum concentration is around $0.1 \%$. Below this limit, pentacene molecules randomly replace $p$-terphenyl molecules with the long axis of the pentacene parallel to the long axis of the displaced host molecule [20, 21]. For crystal growth either co-sublimation [20, 23] or the Bridgman method is widely used $[1,12]$. Both methods require specialist equipment which means that samples of $p$-terphenyl doped with pentacene cannot be prepared in many laboratories.

The resulting single crystal of $p$-terphenyl has three major orientations corresponding to the crystallographic axes, and as was shown by Kim and Weissman, their orientation relative to the external magnetic field, $B_{0}$, is crucial for the signal intensity and the nutation pattern [18]. Thus the orientation of the crystal with respect to the external magnetic field must be carefully adjusted in the spectrometer by TREPR experiments. Indeed, misalignment of the crystal may decimate both the signal intensity and the amplitude of the Torrey oscillations.

From a more practical aspect, single crystals of pentacene in $p$-terphenyl are often employed to align the laser beam in order to optimize photoexcitation of the sample in the microwave resonator. However, this has the disadvantage that the laser beam is directed towards a point sample rather than the extended volume typically occupied by frozen solutions or thin film samples. To avoid this issue, a crushed crystal of pentacene in $p$-terphenyl is sometimes used for laser alignment. This provides a pseudo-powder sample-with essentially no preferred orientation-so that finding a signal is easier. The caveat is that the penetration of light into the crystalline powder is poor, and hence the TREPR signal is relatively weak [7]. By melting and resolidifying a mixture of $p$-terphenyl and pentacene directly within an EPR-tube, a polycrystalline sample is obtained that avoids most of the problems associated with doped single crystals but shows some orientation dependency in the magnetic field.

Here, we present 1,3,5-tri(1-naphtyl)benzene (TNB) as a host matrix for pentacene, see Fig. 1. TNB is a glass-forming compound, which melts around $459 \mathrm{~K}$, and with a glass-transition point at $342 \mathrm{~K}[11,24]$. Not only does pentacene dissolve easily in liquid TNB, but in the absence of oxygen it is also stable at the melting point of TNB. Thus, sample preparation only requires that the two components are mixed together in the desired ratio, melted using a heat gun or oil bath under anaerobic conditions, and then cooled back down to room temperature. Because the shortrange order of the liquid state is maintained in glassy samples, not only is the sample optically transparent but the solute molecules do not become aligned; as typically occurs in crystalline matrices $[15,16]$.

As we show below, not only does this system give rise to a well-resolved TREPR spectrum complete with Torrey oscillations, but more importantly it may be prepared in laboratories equipped with standard equipment [8, 28]. Together, these properties make pentacene dissolved in TNB a suitable test sample for TREPR measurements at room temperature. In addition, it may be used a standard sample 
for calibrating or adjusting the TREPR spectrometer, as well an educational sample for students learning about TREPR spectroscopy.

\section{Results and Discussion}

It is often advantageous to excite chromophores near the maximum optical absorption. The UV-Vis spectrum of pentacene in TNB at room temperature is depicted in Fig. 2. The maximum is close to $590 \mathrm{~nm}$. Nevertheless, there is still reasonable absorption at other widely available wavelengths, such as the second and third harmonics of a Nd:YAG laser, $532 \mathrm{~nm}$ and $355 \mathrm{~nm}$ (see Fig. 2 in SI).

Figure 3 depicts a representative two-dimensional data set of the photoexcited triplet state of pentacene in TNB at room temperature. The spectrum is powder-like and exhibits Torrey oscillations that are $B_{1}$ dependent. Time traces at the absorptive and emissive extrema (Fig. 3, right) are different in their oscillatory behaviour due to the magnetic field dependent dynamics of the system [36]. They follow a Bessel function of zeroth order and contain information about spin relaxation. Using a Fourier transform of the high-field transition starting at the first minimum, the nutation frequency $v_{n}$ was extracted to obtain the $B_{1}$-field following the equation $v_{n}=\sqrt{2} \gamma_{e} B_{1}$, where the factor $\sqrt{2}$ arises due to the $S=1$ nature of the spin system. By recording the kinetic traces as a function of $P_{\mathrm{MW}}$, the frequency of the Torrey oscillations may be related to the square root of the microwave power (see Fig. 1 in SI) and thus the conversion factor, $C$, of the resonator may be determined. In this study, $C$ was found to be $4.7 \mathrm{G} / \sqrt{W}$ which is comparable to the value of $4.2 \mathrm{G} / \sqrt{W}$ specified by Bruker.

A slice through the two-dimensional data set parallel to the magnetic field axis at the time of maximum signal intensity reveals the powder spectrum of the triplet state of pentacene. The zero-field splitting parameters may

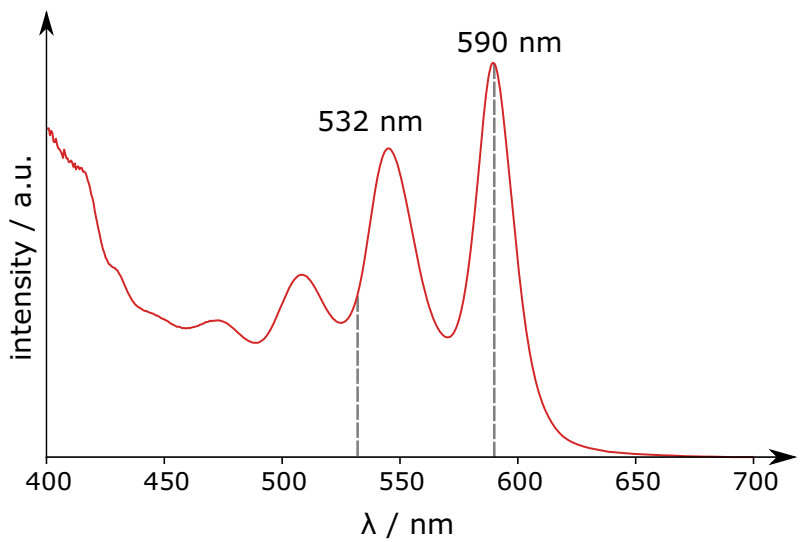

Fig. 2 UV-Vis spectrum of pentacene in TNB glass with indicated positions of the global maximum of the absorption $(590 \mathrm{~nm})$ and the second harmonic $(532 \mathrm{~nm})$ of a Nd:YAG-Laser 

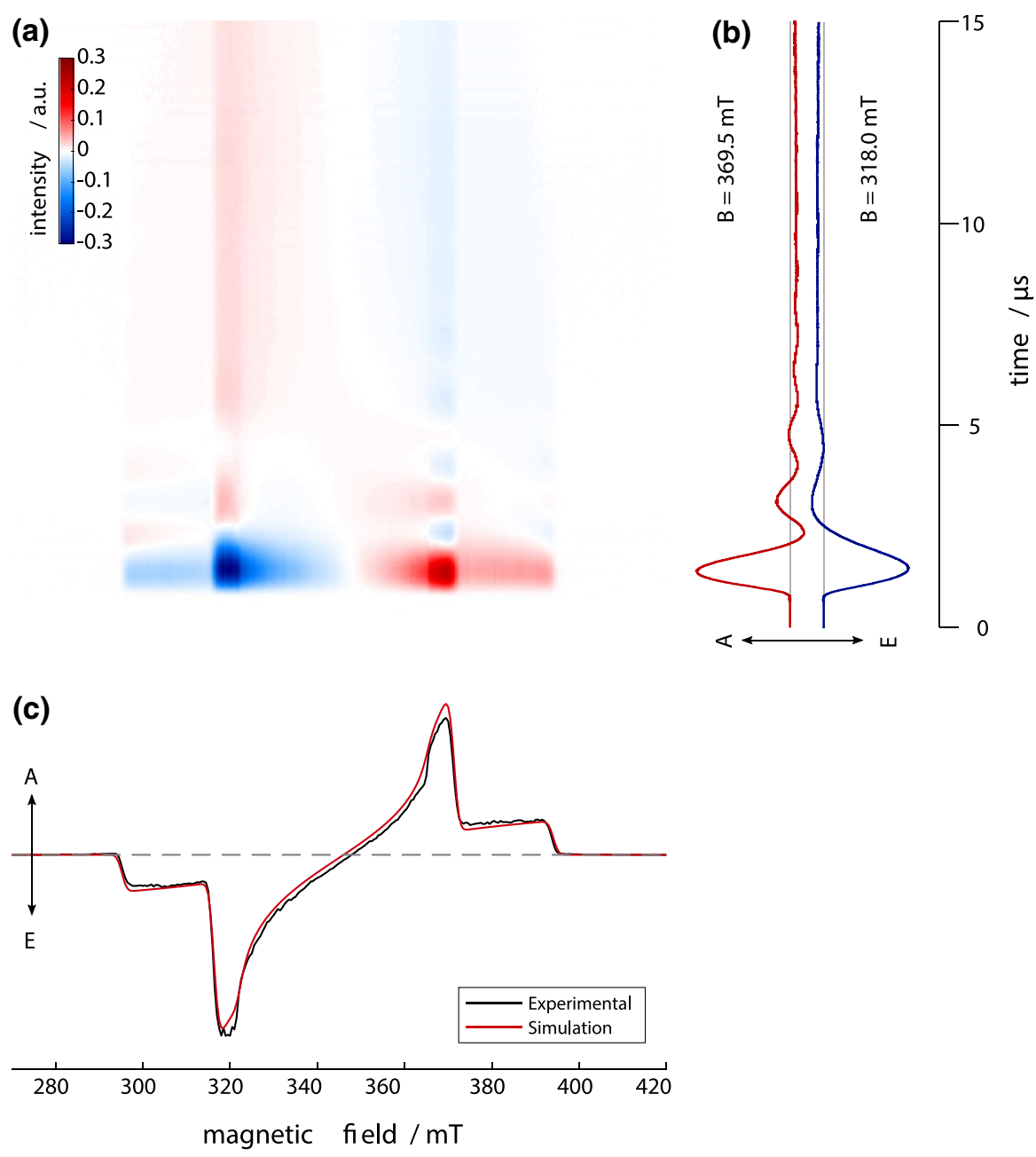

Fig. 3 2D TREPR spectrum of pentacene in TNB with the time traces through the respective extrema (right) and an average over $600 \mathrm{~ns}$ along the magnetic field axis (bottom) centered at $1.4 \mu \mathrm{s}$, excited at $600 \mathrm{~nm}$ together with a simulation

be extracted by simulation $[25,37]$. The values of $|D|=(1385 \pm 4) \mathrm{MHz}$ and $|E|=(50.6 \pm 1.3) \mathrm{MHz}$ are similar to those found for pentacene in $p$-terphenyl crystals [22, 29, 44, 45] and thin films [23]. Surprisingly, the relative populations $p_{1}: p_{2}: p_{3}=0.19: 0.35: 0.46$ differ substantially from literature values of pentacene in $p$-terphenyl $\left(p_{1}: p_{2}: p_{3}=0.08: 0.16: 0.76\right)$ [34, 44], indicating that the environment has a strong effect on the relative rates of intersystem crossing to the three triplet sub-levels.

In contrast to the spectra obtained in single crystals, where the peaks are narrow and depend strongly upon the crystal orientation with respect to the external magnetic field, the powder spectrum stretches over the full $2 D$ range. 
Consequently, there is no need to precisely hit a resonant magnetic field position when using the glassy sample to adjust the laser beam. Moreover, to obtain the essential triplet parameters of the molecule, only a single spectrum needs to be recorded rather than a complete rotation pattern with respect to the three canonical axes. These aspects are helpful not only for teaching TREPR, but also for introducing the concept of a powder pattern, which is generally the case for the TREPR spectra of randomly oriented triplet states in frozen solution.

The TREPR spectra presented here were recorded at room temperature. However, when the temperature is lowered the relaxation rates slow and hence the decay of the spin polarization also slows. We have tested the sample down to $80 \mathrm{~K}$ at which temperature it still gives an intense TREPR spectrum (data not shown).

\section{Conclusion}

We have shown that pentacene dissolved in glassy TNB is a useful TREPR standard. The broad powder-like spectrum and decent signal to noise ratio of the triplet state means that it is well suited for optimizing and characterizing the TREPR spectrometer, adjusting the laser system, and as a (nearly) fail-safe educational sample for beginners in the field.

Due to the ease of preparation and powder-like TREPR spectrum, pentacene in TNB is clearly superior to a single crystal of pentacene in $p$-terphenyl as a standard sample. Finally, because TNB is glass-forming and readily dissolves non-polar substances, it may turn out to be a convenient and useful alternative host matrix for TREPR spectroscopy of other aromatic molecules at room temperature, and find use in other applications, such as zero-field room-temperature masers [9, 10, 30, 32].

\section{Experimental}

\subsection{Sample Preparation}

A sample consisting of $0.01 \mathrm{wt} \%$ pentacene as prepared by literature methods [31] in 1,3,5-tri(1-naphthyl)benzene (TCI Germany) was prepared by mixing the two powders and grinding in a mortar. The sample was placed in a synthetic-quartz EPR-tube (3 mm inner diameter, QSIL Ilmasil), evacuated and flushed with argon several times. Subsequently, the mixture was heated to $195^{\circ} \mathrm{C}$ under argon using an oil bath, at which temperature it forms a homogenous dark red liquid. The tube was removed from the oil bath and allowed to cool to ambient temperature, leading to the formation of a glass. The EPR-tube was evacuated at room temperature and flame sealed. Occasionally, the sample crystallizes, but the glass can easily be regenerated by melting the sample and subsequently cooling again. 


\subsection{UV-Vis Spectroscopy}

A ground-up mixture of approximately $0.1 \mathrm{wt} \%$ pentacene and TNB was placed in a quartz cuvette with $1 \mathrm{~mm}$ path length and melted using an oil bath at $195{ }^{\circ} \mathrm{C}$. After vitrification by allowing the sample to cool to ambient temperature, the glassy sample was measured on a Specord 210 Plus (Analytik Jena, Germany) in the range from 350 to $800 \mathrm{~nm}$. A background correction was performed by subtracting the spectrum of the pure glassy matrix which was measured under the same conditions.

\subsection{Time Resolved EPR Spectroscopy}

TREPR spectra were recorded using a Bruker EMX spectrometer with a Bruker EMX premiumX microwave bridge and a dielectric-ring resonator (Bruker ER 4118X-MD5) which was placed in a closed-cycle cryostat (Cryogenic CF VTC) in a helium atmosphere. Time dependent signals were acquired using a digital oscilloscope (Teledyne LeCroy HDO9204) with a digitizing rate of $2 \mathrm{~ns} / 8$ bit.

Pulsed optical excitation was provided by an optical parametric oscillator (OPO) system (GWU primoScan/BB/120-INDI) pumped by a Nd:YAG laser (SpectraPhysics Quanta-Ray INDI PS 51/52) with a pulse width of approximately $6 \mathrm{~ns}$, a pulse energy of $1.5 \mathrm{~mJ}$ and a shot repetition rate of $20 \mathrm{~Hz}$. Each magnetic field point was recorded 100 times, wavelength dependent spectra were recorded with $1.0 \mathrm{~mW}$ microwave power and subsequently normalised to the same number of photons.

Processing and analysis of the time traces were performed with self-written MATLAB ${ }^{\circledR}$-routines. Simulation of the spectrum averaged over the maximum signal was performed using the Tsim-programme [25] which is based on the EasySpin [37] package for MATLAB ${ }^{\circledR}$.

Supplementary Information The online version supplementary material available at https://doi.org/10. 1007/s00723-021-01420-4.

Acknowledgements The authors thank Yan Fett and PD Dr. Till Biskup (Saarland University) for interfacing the Teledyne LeCroy Oscilloscope and the EMX Spectrometer and Saarland University for financial support.

Funding Open Access funding enabled and organized by Projekt DEAL.

\section{Declaration}

Conflict of interest The authors declare that they have no conflict of interest.

Open Access This article is licensed under a Creative Commons Attribution 4.0 International License, which permits use, sharing, adaptation, distribution and reproduction in any medium or format, as long as you give appropriate credit to the original author(s) and the source, provide a link to the Creative Commons licence, and indicate if changes were made. The images or other third party material in this article are included in the article's Creative Commons licence, unless indicated otherwise in a credit line to the material. If material is not included in the article's Creative Commons licence and your intended use is not permitted by statutory regulation or exceeds the permitted use, you will need to obtain permission 
directly from the copyright holder. To view a copy of this licence, visit http://creativecommons.org/licen ses/by/4.0/.

\section{References}

1. Q. Ai, P. Chen, Y. Feng, Y. Xu, Growth of pentacene-doped p-terphenyl crystals by vertical bridgman technique and doping effect on their characterization. Cryst. Growth Des. 17(5), 24732477 (2017). https://doi.org/10.1021/acs.cgd.6b01900

2. A. Bertran, K.B. Henbest, M. De Zotti, M. Gobbo, C.R. Timmel, M. Di Valentin, A.M. Bowen, Light-induced triplet-triplet electron resonance spectroscopy. J. Phys. Chem. Lett. 12(1), 80-85 (2021). https://doi.org/10.1021/acs.jpclett.0c02884

3. A. Bieber, D. Bücker, M. Drescher, Light-induced dipolar spectroscopy-a quantitative comparison between LiDEER and LaserIMD. J. Magn. Reson. 296, 29-35 (2018). https://doi.org/10. 1016/j.jmr.2018.08.006

4. T. Biskup, Structure-function relationship of organic semiconductors: detailed insights from time-resolved EPR spectroscopy. Front. Chem. 7, 1-22 (2019). https://doi.org/10.3389/fchem. 2019.00010

5. T. Biskup, Doping of organic semiconductors: insights from EPR spectroscopy. Appl. Phys. Lett. 119, 010503 (2021). https://doi.org/10.1063/5.0054685

6. R. Bittl, S. Weber, Transient radical pairs studied by time-resolved EPR. Biochim. Biophys. Acta 1707, 117-126 (2005). https://doi.org/10.1016/j.bbabio.2004.03.012

7. S. Bogatko, P.D. Haynes, J. Sathian, J. Wade, J.S. Kim, K.J. Tan, J. Breeze, E. Salvadori, A. Horsfield, M. Oxborrow, Molecular design of a room-temperature maser. J. Phys. Chem. C 120(15), 8251-8260 (2016). https://doi.org/10.1021/acs.jpcc.6b00150

8. P.A. Bonvallet, C.J. Breitkreuz, Y.S. Kim, E.M. Todd, K. Traynor, C.G. Fry, M.D. Ediger, R.J. McMahon, Organic glass-forming materials: 1,3,5-tris(naphthyl)benzene derivatives. J. Org. Chem. 72(26), 10051-10057 (2007). https://doi.org/10.1021/jo701921m

9. J. Breeze, K.J. Tan, B. Richards, J. Sathian, M. Oxborrow, N.M. Alford, Enhanced magnetic Purcell effect in room-temperature masers. Nat. Commun. 6(1), 6215 (2015). https://doi.org/10. 1038/ncomms 7215

10. J.D. Breeze, E. Salvadori, J. Sathian, N.M. Alford, C.W.M. Kay, Room-temperature cavity quantum electrodynamics with strongly coupled Dicke states. npj Quantum Inf. 3(1), 40 (2017). https://doi.org/10.1038/s41534-017-0041-3

11. S. Capaccioli, G. Ruocco, F. Zamponi, Dynamically correlated regions and configurational entropy in supercooled liquids. J. Phys. Chem. B 112(34), 10652-10658 (2008). https://doi.org/ 10.1021/jp802097u

12. S. Cui, Y. Liu, G. Li, Q. Han, C. Ge, L. Zhang, Q. Guo, X. Ye, X. Tao, Growth regulation of pentacene-doped p-terphenyl crystals on their physical properties for promising maser gain medium. Cryst. Growth Des. 20(2), 783-792 (2020). https://doi.org/10.1021/acs.cgd.9b01190

13. G.R. Eaton, S.S. Eaton, D.P. Barr, R.T. Weber, Quantitative EPR (Springer Vienna, Vienna, 2010). https://doi.org/10.1007/978-3-211-92948-3

14. M.D. Forbes, L.E. Jarocha, S. Sim, V.F. Tarasov, Time-Resolved Electron Paramagnetic Resonance Spectroscopy: History, Technique, and Application to Supramolecular and Macromolecular Chemistry, vol. 47 (Elsevier Ltd., Amsterdam, 2013). https://doi.org/10.1016/B978-0-12407754-6.00001-6

15. C.W.M. Kay, M. Di Valentin, K. Möbius, A time-resolved Electron Nuclear Double Resonance (ENDOR) study of the photoexcited triplet state of free-base tetraphenylporphyrin. Sol. Energy Mater. Sol. Cells 38(1-4), 111-118 (1995). https://doi.org/10.1016/0927-0248(94)00219-3

16. C.W.M. Kay, M. Di Valentin, K. Möbius, Time-resolved EPR and ENDOR study of the photoexcited triplet state of free-base tetraphenylchlorin in a crystalline toluene matrix. J Chem Soc Perkin Trans 2(12), 2563-2568 (1997). https://doi.org/10.1039/a702468a

17. S.S. Kim, S.I. Weissman, Detection of transient electron paramagnetic resonance. J. Magn. Reson. (1969) 24(1), 167-169 (1976). https://doi.org/10.1016/0022-2364(76)90243-2 
18. S.S. Kim, S.I. Weissman, Transient magnetization following photoexcitation. Rev. Chem. Intermed. 3(1-2), 107-120 (1979). https://doi.org/10.1007/BF03052283

19. S.Z. Kiss, A.M. Rostas, L. Heidinger, N. Spengler, M.V. Meissner, N. MacKinnon, E. Schleicher, S. Weber, J.G. Korvink, A microwave resonator integrated on a polymer microfluidic chip. J. Magn. Reson. 270, 169-175 (2016). https://doi.org/10.1016/j.jmr.2016.07.008

20. J. Köhler, A.C. Brouwer, E.J. Groenen, J. Schmidt, On the intersystem crossing of pentacene in p-terphenyl. Chem. Phys. Lett. 250(1), 137-144 (1996). https://doi.org/10.1016/0009-2614(95) 01393-8

21. J. Lang, D.J. Sloop, T. Lin, Mapping the molecular axes of pentacene- d14 doped in p -terphenyl single crystal using pulsed EPR technique in near zero magnetic field. Mol. Phys. 104(10-11), 1643-1648 (2006). https://doi.org/10.1080/00268970500510608

22. T. Lin, Novel pulsed electron paramagnetic resonance techniques for the studies of structure and dynamics of photo-excited triplet state of organic molecules: a professional journey. J. Chin. Chem. Soc. 65(2), 163-188 (2018). https://doi.org/10.1002/jccs.201700120

23. D. Lubert-Perquel, E. Salvadori, M. Dyson, P.N. Stavrinou, R. Montis, H. Nagashima, Y. Kobori, S. Heutz, C.W.M. Kay, Identifying triplet pathways in dilute pentacene films. Nat. Commun. 9(1), 4222 (2018). https://doi.org/10.1038/s41467-018-06330-X

24. J.H. Magill, D.J. Plaze, Crystallization kinetics of 1:3:5 Tri- $\alpha$-naphthyl benzene. Nature 209(5018), 70-71 (1966). https://doi.org/10.1038/209070a0

25. D.L. Meyer, F. Lombeck, S. Huettner, M. Sommer, T. Biskup, Direct S0 $\rightarrow$ T excitation of a conjugated polymer repeat unit: unusual spin-forbidden transitions probed by time-resolved electron paramagnetic resonance spectroscopy. J. Phys. Chem. Lett. 8(7), 1677-1682 (2017). https://doi.org/ 10.1021/acs.jpclett.7b00644

26. D.L. Meyer, R. Matsidik, D. Fazzi, M. Sommer, T. Biskup, Probing exciton delocalization in organic semiconductors: insight from time-resolved electron paramagnetic resonance and magnetophotoselection experiments. J. Phys. Chem. Lett. 9(24), 7026-7031 (2018). https://doi.org/10.1021/ acs.jpclett.8b03196

27. S.K. Misra, Quantum computing/quantum information processing in view of electron magnetic/ electron paramagnetic resonance technique/spectroscopy. in Electron Spin Resonance (ESR) Based Quantum Computing (Springer, 2016), pp. 1-23. https://doi.org/10.1007/978-1-4939-3658-8_1

28. S. Nitnara, A comparison of the glass former 1,3,5-tri(Naphthyl)benzene and the crystalline p-terphenyl hosts for pentacene masers. Ph.D. thesis, Imperial College London (2020). https://doi.org/10. $25560 / 87525$

29. J.L. Ong, D.J. Sloop, T. Lin, Temperature dependence studies of the paramagnetic properties of the photoexcited triplet states of pentacene in p-terphenyl, benzoic acid, and naphthalene crystals. J. Phys. Chem. 97(30), 7833-7838 (1993). https://doi.org/10.1021/j100132a008

30. M. Oxborrow, J.D. Breeze, N.M. Alford, Room-temperature solid-state maser. Nature 488(7411), 353-356 (2012). https://doi.org/10.1038/nature11339

31. C. Pramanik, G.P. Miller, An improved synthesis of pentacene: rapid access to a benchmark organic semiconductor. Molecules 17(4), 4625-4633 (2012). https://doi.org/10.3390/molecules17044625

32. E. Salvadori, J.D. Breeze, K.J. Tan, J. Sathian, B. Richards, M.W. Fung, G. Wolfowicz, M. Oxborrow, N.M. Alford, C.W.M. Kay, Nanosecond time-resolved characterization of a pentacene-based room-temperature MASER. Sci. Rep. 7(1), 41836 (2017). https://doi.org/10.1038/srep41836

33. S. Siegel, H.S. Judeikis, A magnetophotoselection study of the polarizations of the absorption bands of some structurally related hydrocarbons and heterocyclic molecules. J. Phys. Chem. 70(7), 22052211 (1966). https://doi.org/10.1021/j100879a021

34. D.J. Sloop, H. Yu, T. Lin, S.I. Weissman, Electron spin echoes of a photoexcited triplet: pentacene in p-terphenyl crystals. J. Chem. Phys. 75(8), 3746-3757 (1981). https://doi.org/10.1063/1.442520

35. M.B. Smith, J. Michl, Singlet fission. Chem. Rev. 110(11), 6891-6936 (2010). https://doi.org/10. $1021 / \mathrm{cr} 1002613$

36. D. Stehlik, C.H. Bock, M.C. Thurnauer, Transient Epr-Spectroscopy of Photoinduced Electronic Spin States in Rigid Matrices (Elsevier, Amsterdam, 1989). https://doi.org/10.1016/b978-0-44488050-5.50016-1

37. S. Stoll, A. Schweiger, EasySpin, a comprehensive software package for spectral simulation and analysis in EPR. J. Magn. Reson. 178(1), 42-55 (2006). https://doi.org/10.1016/j.jmr.2005.08.013

38. C.E. Tait, P. Neuhaus, H.L. Anderson, C.R. Timmel, D. Carbonera, M. Di Valentin, HYSCORE on photoexcited triplet states. Appl. Magn. Reson. 46(4), 389-409 (2015). https://doi.org/10.1007/ s00723-014-0624-5 
39. M.C. Thurnauer, J.R. Norris, Magnetophotoselection applied to the triplet state observed by EPR in photosynthetic bacteria. Biochem. Biophys. Res. Commun. 73(2), 501-506 (1976). https://doi.org/ 10.1016/0006-291X(76)90735-X

40. H.C. Torrey, Transient nutations in nuclear magnetic resonance. Phys. Rev. 76(8), 1059-1068 (1949). https://doi.org/10.1103/PhysRev.76.1059

41. S. Weber, Transient EPR. in eMagRes, vol. 6( John Wiley \& Sons, Ltd, Chichester, 2017), pp. 255270. https://doi.org/10.1002/9780470034590.emrstm1509

42. L.R. Weiss, S.L. Bayliss, F. Kraffert, K.J. Thorley, J.E. Anthony, R. Bittl, R.H. Friend, A. Rao, N.C. Greenham, J. Behrends, Strongly exchange-coupled triplet pairs in an organic semiconductor. Nat. Phys. 13(2), 176-181 (2017). https://doi.org/10.1038/nphys3908

43. J. Xu, L.E. Jarocha, T. Zollitsch, M. Konowalczyk, K.B. Henbest, S. Richert, M.J. Golesworthy, J. Schmidt, V. Déjean, D.J. Sowood, M. Bassetto, J. Luo, J.R. Walton, J. Fleming, Y. Wei, T.L. Pitcher, G. Moise, M. Herrmann, H. Yin, H. Wu, R. Bartölke, S.J. Käsehagen, S. Horst, G. Dautaj, P.D. Murton, A.S. Gehrckens, Y. Chelliah, J.S. Takahashi, K.W. Koch, S. Weber, I.A. Solov'yov, C. Xie, S.R. Mackenzie, C.R. Timmel, H. Mouritsen, P.J. Hore, Magnetic sensitivity of cryptochrome 4 from a migratory songbird. Nature 594(7864), 535-540 (2021). https://doi.org/10.1038/ s41586-021-03618-9

44. T.C. Yang, D.J. Sloop, S.I. Weissman, T. Lin, Zero-field magnetic resonance of the photo-excited triplet state of pentacene at room temperature. J. Chem. Phys. 113(24), 11194-11201 (2000). https:// doi.org/10.1063/1.1326069

45. H.L. Yu, T. Lin, S.I. Weissman, D.J. Sloop, Time resolved studies of pentacene triplets by electron spin echo spectroscopy. J. Chem. Phys. 80(1), 102-107 (1984). https://doi.org/10.1063/1.446491

Publisher's Note Springer Nature remains neutral with regard to jurisdictional claims in published maps and institutional affiliations. 